\title{
Metal and organo-catalysed asymmetric hydroaminomethylation of styrenes
}

\author{
Barbara Villa-Marcos, Jianliang Xiao* \\ Department of Chemistry, University of Liverpool, Liverpool L69 7ZD, UK
}

\section{A R T I C L E I N F O}

Article history:

Received 12 September 2014

Accepted 25 October 2014

Published 20 January 2015

\section{Keywords:}

Asymmetric hydroaminomethylation

Hydroformylation

Chiral amine

Metal catalysis

Organocatalysis

\section{A B S T R A C T}

A new protocol that enables asymmetric hydroaminomethylation of styrenes to afford chiral amines has been developed. Catalysed by an Rh-phosphine species and a chiral phosphoric acid, styrenes are converted into $\beta$-chiral amines with good enantioselectivities under syngas in the presence of an amine and Hantzsch ester. The reaction involves two key steps, hydroformylation and reductive amination, with the former catalysed by the Rh species whilst the latter by the phosphoric acid.

(C) 2015, Dalian Institute of Chemical Physics, Chinese Academy of Sciences. Published by Elsevier B.V. All rights reserved.

\section{Introduction}

We recently reported that imino bonds can be reduced highly enantioselectively under metal-organo cooperative catalysis [1-5]. Since olefins such as styrenes could be selectively hydroformylated to the corresponding $\alpha$-branched aldehydes [6], which readily condense with an amine to afford imines, it became possible to us that $\beta$-chiral amines might be accessible via a similar strategy (Scheme 1). The enantioselectivity would be achieved through a dynamic kinetic resolution (DKR) process preceding the reduction. In the presence of a chiral acid catalyst, imines are expected to undergo fast racemisation by tautomerisation, and one of the enantiomers of the iminium cation could be selectively reduced, leading to chiral amines [7].

Hydroformylation of an alkene, followed by reductive amination of the resulting aldehyde intermediate with an amine, is known as hydroaminomethylation [8]. It represents a one-pot, atom-efficient reaction for the synthesis of amines, one of the most ubiquitous functionalities in chemical synthesis. A suita- ble catalyst for hydroaminomethylation must fulfil a number of requirements (Scheme 2). It must be highly regioselective to either the linear or branched aldehyde, depending on the desired final product. The catalyst must be active for the enamine/imine hydrogenation, as a slow hydrogenation leads to aldol-type side reactions [9]. Finally, the catalyst must be selective for enamine/imine hydrogenation over hydrogenation of aldehydes. In addition, the enamine/imine isomerisation must be faster than the subsequent hydrogenation to ensure efficient DKR.

The first example of hydroaminomethylation was reported by Reppe et al. at BASF in the early 1950s [10]. Simple alkenes, like ethene or propene, were converted to secondary and tertiary amines in low yields, with ammonia under harsh conditions of up to $390{ }^{\circ} \mathrm{C}$ and 950 bar $\mathrm{H}_{2}$ using [Fe(CO)5] in almost stoichiometric quantity. Significant progress has been made since, with notable contributions being made by the groups of Eilbracht and Beller [8,11-13]. There are, however, few studies concerning asymmetric hydroaminomethylation, with none

* Corresponding author. Tel: +44-1517942937; E-mail: jxiao@liv.ac.uk 


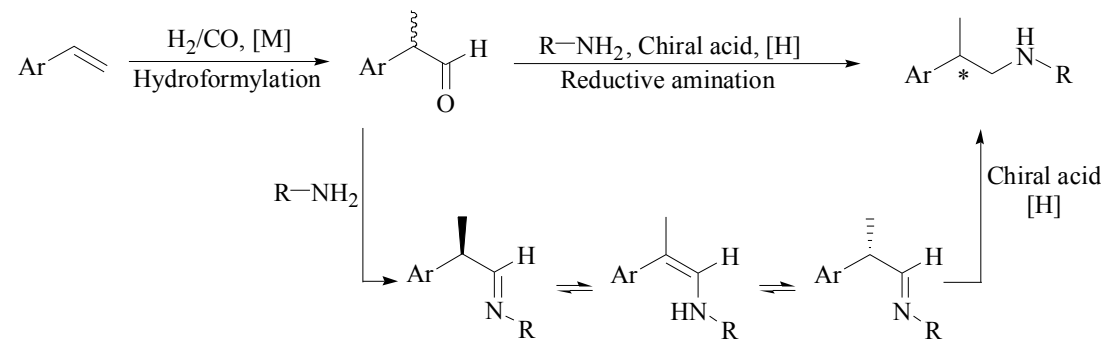

Scheme 1. Asymmetric hydroaminomethylation via tandem hydroformylation-asymmetric reductive amination.

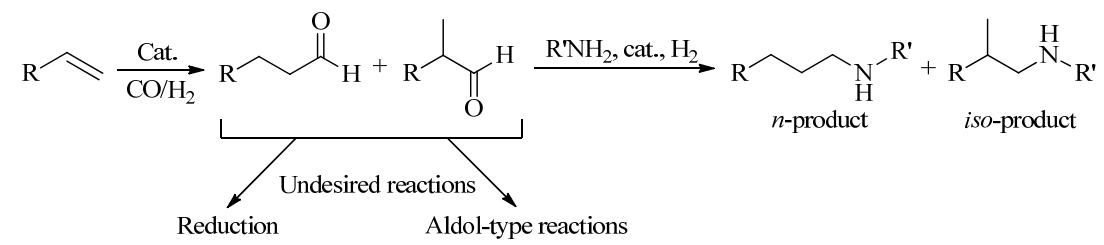

Scheme 2. Chemo- and $i / n$ regio-selectivity issues in the hydroaminomethylation sequence.

reporting significant enantioselectivities [14,15].

\section{Experimental}

\subsection{General procedure for the synthesis of racemic mixtures of $1 a-p$}

To a glass liner equipped with a stir bar was added styrene (5 mmol), [Rh(acac)(CO) 2 ] $(25 \mu \mathrm{mol}),\left(4-\mathrm{MeOC}_{6} \mathrm{H}_{4}\right)_{3} \mathrm{P} \quad(50$ $\mu \mathrm{mol})$, and toluene $(2 \mathrm{~mL})$. The glass liner was then placed into an autoclave, followed by degassing with syngas three times. The reaction was carried out at 11 bar syngas with stirring at $50{ }^{\circ} \mathrm{C}$ overnight. The stirring was then stopped, and the autoclave allowed to cool down to room temperature. The syngas was then carefully released in a fume hood and the solution was filtered through celite, transferred to a flask, and concentrated to afford the crude product. Flash chromatography purification with a column of silica gel eluted with petroleum ether/ethyl acetate (50/1) yielded the desired aldehyde product.

To an oven-dried Schlenk tube equipped with a stir bar was added the aldehyde prepared $(0.5 \mathrm{mmol})$, amine $(0.5 \mathrm{mmol})$ and an Iridicycle catalyst ( $5 \mu \mathrm{mol})$ [25]. The tube was degassed with nitrogen three times. $\mathrm{MeOH}(4 \mathrm{~mL})$ was then added with syringe, followed by $\mathrm{HCOOH} / \mathrm{Et}_{3} \mathrm{~N}(5: 2,1 \mathrm{~mL})$. The resulting mixture was stirred at $80{ }^{\circ} \mathrm{C}$ for $3 \mathrm{~h}$. The stirring was then stopped, and the reaction mixture allowed to cool down to room temperature. The reaction was then quenched with water and basified with saturated $\mathrm{KOH}_{\mathrm{aq}}$ solution, extracted with ethyl acetate and dried over $\mathrm{MgSO}_{4}$. Flash chromatography purification with a column of silica gel eluted with petroleum ether/ethyl acetate (15/1) yielded the racemic mixtures of 1a-p.

\subsection{General procedure for asymmetric hydroaminomethylation}

To a glass liner equipped with a stir bar was added 4 Å MS (100 mg), alkene $(0.4 \mathrm{mmol})$, amine $(0.25 \mathrm{mmol})$, $\left[\mathrm{Rh}(\mathrm{acac})(\mathrm{CO})_{2}\right] \quad(1.25 \mu \mathrm{mol}),\left(4-\mathrm{MeO}-\mathrm{C}_{6} \mathrm{H}_{4}\right)_{3} \mathrm{P} \quad(2.5 \mu \mathrm{mol})$
Et-HEH (0.6 mol), TRIP (12.5 $\mu \mathrm{mol})$, and toluene $(2 \mathrm{~mL})$. The glass liner was then placed into an autoclave, followed by degassing with syngas three times. The reaction was carried out at 11 bar syngas with stirring at $50{ }^{\circ} \mathrm{C}$ for $3 \mathrm{~d}$. The stirring was then stopped, and the autoclave allowed to cool down to room temperature. The syngas was then carefully released in a fume hood and the solution was filtered through celite, transferred to a flask, and concentrated to afford the crude product. Flash chromatography purification with a column of silica gel eluted with petroleum ether/ethyl acetate (40/1 to $30 / 1)$ yielded the desired amine product.

\subsection{Analytical data}

4-Methoxy- $N$-(2-p-tolylpropyl)aniline, 1a [17]. The product (50 mg, 79\% yield, $80 \%$ ee) was obtained as a colourless oil according to the general procedure in $3 \mathrm{~d}$; ${ }^{1} \mathrm{H}$ NMR $(400 \mathrm{MHz}$, $\left.\mathrm{CDCl}_{3}\right) \delta 1.30(\mathrm{~d}, J=7.0 \mathrm{~Hz}, 3 \mathrm{H}), 2.33(\mathrm{~s}, 3 \mathrm{H}), 2.98-3.05(\mathrm{~m}, 1 \mathrm{H})$, 3.16 (dd, $A$ of $\left.A B X, J_{A B}=12.1 \mathrm{~Hz}, J_{\mathrm{AX}}=8.4 \mathrm{~Hz}, 1 \mathrm{H}\right), 3.28(\mathrm{dd}, \mathrm{B}$ of $\left.\mathrm{ABX}, J_{\mathrm{AB}}=12.1 \mathrm{~Hz}, J_{\mathrm{BX}}=6.2 \mathrm{~Hz}, 1 \mathrm{H}\right), 3.74(\mathrm{~s}, 3 \mathrm{H}), 3.53-6.57(\mathrm{~m}$, $2 \mathrm{H}), 6.74-6.78(\mathrm{~m}, 2 \mathrm{H}), 7.10-7.15(\mathrm{~m}, 4 \mathrm{H}) ;{ }^{13} \mathrm{C} \mathrm{NMR}(100 \mathrm{MHz}$, $\left.\mathrm{CDCl}_{3}\right) \delta 20.0,21.1,38.8,52.1,55.8,114.4,114.9,127.2,129.4$, 136.1, 141.6, 142.4, 152.1; $\mathrm{C}_{17} \mathrm{H}_{22} \mathrm{NO}[\mathrm{M}+\mathrm{H}]^{+}: \mathrm{m} / z$ Calcd.: 256.1701; Found: 256.1706; HPLC (Chiralcel OJ, hexane:isopropanol $=90: 10$, flow rate $0.5 \mathrm{~mL} / \mathrm{min}, \lambda=254 \mathrm{~nm}): t_{\mathrm{R}}=16.5$ $\min$ (minor), $t_{\mathrm{R}}=18.7$ min (major).

3-Methoxy- $N$-(2-p-tolylpropyl)aniline, 1b. The product (53 $\mathrm{mg}, 83 \%$ yield, $84 \%$ ee) was obtained as a colourless oil according to the general procedure in $3 \mathrm{~d} ;{ }^{1} \mathrm{H}$ NMR $(400 \mathrm{MHz}$, $\left.\mathrm{CDCl}_{3}\right) \delta 1.31(\mathrm{~d}, J=6.9 \mathrm{~Hz}, 3 \mathrm{H}), 2.33(\mathrm{~s}, 3 \mathrm{H}), 2.97-3.06(\mathrm{~m}, 1 \mathrm{H})$, 3.19 (dd, A of $\left.\mathrm{ABX}, J_{\mathrm{AB}}=12.2 \mathrm{~Hz}, J_{\mathrm{AX}}=8.3 \mathrm{~Hz}, 1 \mathrm{H}\right), 3.30(\mathrm{dd}, \mathrm{B}$ of $\left.\mathrm{ABX}, J_{\mathrm{AB}}=12.2 \mathrm{~Hz}, J_{\mathrm{BX}}=6.2 \mathrm{~Hz}, 1 \mathrm{H}\right), 3.75(\mathrm{~s}, 3 \mathrm{H}), 6.13(\mathrm{t}, J=2.1$ $\mathrm{Hz}, 1 \mathrm{H}$ ), 6.18 (dd, $J=8.1,2.1 \mathrm{~Hz}, 1 \mathrm{H}$ ), 8.25 (dd, $J=8.1,2.1 \mathrm{~Hz}$, 1H), $7.05(\mathrm{t}, J=8.1 \mathrm{~Hz}, 1 \mathrm{H}), 7.10-7.15(\mathrm{~m}, 4 \mathrm{H}) ;{ }^{13} \mathrm{C}$ NMR $(100$ $\left.\mathrm{MHz}, \mathrm{CDCl}_{3}\right) \delta 18.5,21.0,38.8,50.9,55.1,98.8,102.4,106.1$, 127.1, 129.3, 129.9, 136.1, 141.4, 149.5, 160.8; HPLC (Chiralcel OD-H, hexane:isopropanol $=98: 2$, flow rate $0.5 \mathrm{~mL} / \mathrm{min}, \lambda=$ $254 \mathrm{~nm}$ ): $t_{\mathrm{R}}=30.1 \mathrm{~min}$ (minor), $t_{\mathrm{R}}=37.2 \mathrm{~min}$ (major).

3 -Methyl- $N$-(2- $p$-tolylpropyl)aniline, 1c. The product (52 
mg, $87 \%$ yield, $78 \%$ ee) was obtained as a clear yellow oil according to the general procedure in $3 \mathrm{~d}$; ${ }^{1} \mathrm{H}$ NMR $(400 \mathrm{MHz}$, $\left.\mathrm{CDCl}_{3}\right) \delta 1.31(\mathrm{~d}, J=7.0 \mathrm{~Hz}, 3 \mathrm{H}), 2.26(\mathrm{~s}, 3 \mathrm{H}), 2.34(\mathrm{~s}, 3 \mathrm{H})$, 2.97-3.05 (m, 1H), 3.19 (dd, A of ABX, $J_{\mathrm{AB}}=12.2 \mathrm{~Hz}, J_{\mathrm{AX}}=8.3 \mathrm{~Hz}$, $1 \mathrm{H}$ ), 3.31 (dd, B of $A B X, J_{A B}=12.2 \mathrm{~Hz}, J_{\mathrm{BX}}=6.2 \mathrm{~Hz}, 1 \mathrm{H}$ ), 3.52 (brs, $1 \mathrm{H}), 6.38-6.39(\mathrm{~m}, 2 \mathrm{H}), 6.51(\mathrm{~d}, J=7.4 \mathrm{~Hz}, 1 \mathrm{H}), 7.02-7.06(\mathrm{~m}$, $1 \mathrm{H}), 7.11-7.15(\mathrm{~m}, 4 \mathrm{H})$; ${ }^{13} \mathrm{C}$ NMR $\left(100 \mathrm{MHz}, \mathrm{CDCl}_{3}\right) \delta 20.0,21.2$, 21.8, 39.0, 51.1, 110.2, 113.9, 118.4, 127.3, 129.2, 129.5, 136.2, 139.0, 141.7, 148.4; HRMS for $\mathrm{C}_{17} \mathrm{H}_{22} \mathrm{~N}[\mathrm{M}+\mathrm{H}]^{+}: \mathrm{m} / z$ Calcd.: 240.1747; Found: 240.1744; HPLC (Chiralcel OD-H, hexane: isopropanol $=99.8: 0.2$, flow rate $1 \mathrm{~mL} / \mathrm{min}, \lambda=254 \mathrm{~nm}): t_{\mathrm{R}}=$ $20.2 \mathrm{~min}$ (minor), $t_{\mathrm{R}}=20.9 \mathrm{~min}$ (major).

2-Methyl- $N$-(2- $p$-tolylpropyl)aniline, 1d. The product (36 mg, $61 \%$ yield, $86 \%$ ee) was obtained as a colourless oil according to the general procedure in $3 \mathrm{~d}$; ${ }^{1} \mathrm{H}$ NMR $(400 \mathrm{MHz}$, $\left.\mathrm{CDCl}_{3}\right) \delta 1.34(\mathrm{~d}, J=6.9 \mathrm{~Hz}, 3 \mathrm{H}), 1.93(\mathrm{~s}, 3 \mathrm{H}), 2.33(\mathrm{~s}, 3 \mathrm{H})$, 3.04-3.09 (m, 1H), 3.19 (dd, A of ABX, $J_{\mathrm{AB}}=11.9 \mathrm{~Hz}, J_{\mathrm{AX}}=8.4 \mathrm{~Hz}$, $1 \mathrm{H}$ ), 3.37 (dd, B of $A B X, J_{A B}=12.2 \mathrm{~Hz}, J_{\mathrm{BX}}=5.8 \mathrm{~Hz}, 1 \mathrm{H}$ ), 3.43 (brs, $1 \mathrm{H}), 6.64$ (d, $J=7.7 \mathrm{~Hz}, 2 \mathrm{H}), 7.00$, (d, $J=7.4 \mathrm{~Hz}, 1 \mathrm{H}), 7.10-7.12$ $(\mathrm{m}, 5 \mathrm{H}) ;{ }^{13} \mathrm{C} \mathrm{NMR}\left(100 \mathrm{MHz}, \mathrm{CDCl}_{3}\right) \delta 17.2,19.6,21.0,38.7$, 51.0, 109.9, 116.8, 122.1, 127.1, 129.4, 129.5, 130.0, 136.2, 141.4, 146.1; HRMS for $\mathrm{C}_{17} \mathrm{H}_{22} \mathrm{~N}$ [M+H]+: $m / z$ Calcd.: 240.1747; Found: 240.1746; HPLC (Chiralcel OD-H, hexane:isopropanol = 99:1, flow rate $1 \mathrm{~mL} / \mathrm{min}, \lambda=254 \mathrm{~nm}): t_{\mathrm{R}}=7.2 \mathrm{~min}($ major $), t_{\mathrm{R}}=$ 7.9 min (minor).

4-Bromo- $N$-(2- $p$-tolylpropyl)aniline, 1e. The product (34 $\mathrm{mg}, 45 \%$ yield, $79 \%$ ee) was obtained as a clear oil according to the general procedure in $3 \mathrm{~d}$; ${ }^{1} \mathrm{H}$ NMR $\left(400 \mathrm{MHz}^{\mathrm{CDCl}} 3\right) \delta 1.30$ (d, $J=7.0 \mathrm{~Hz}, 3 \mathrm{H}$ ), 2.33 (s, 3H), 2.95-3.03 (m, 1H), 3.15 (dd, A of $\left.\mathrm{ABX}, J_{\mathrm{AB}}=12.3 \mathrm{~Hz}, J_{\mathrm{AX}}=8.5 \mathrm{~Hz}, 1 \mathrm{H}\right), 3.28\left(\mathrm{dd}, \mathrm{B}\right.$ of $\mathrm{ABX}, J_{\mathrm{AB}}=$ $\left.12.3 \mathrm{~Hz}, J_{\mathrm{BX}}=6.0 \mathrm{~Hz}, 1 \mathrm{H}\right), 3.57$ (brs, 1H), 6.41-6.44 (m, 2H), 7.08-7.14 (m, 4H), 7.19-7.23 (m, 2H); ${ }^{13} \mathrm{C}$ NMR (100 MHz, $\left.\mathrm{CDCl}_{3}\right) \delta 19.8,21.0,38.7,50.9,108.8,114.5,127.5,129.4,131.9$, 136.3, 141.1, 147.1; $\mathrm{C}_{16} \mathrm{H}_{19}{ }^{79} \mathrm{BrN}[\mathrm{M}+\mathrm{H}]^{+}: \mathrm{m} / z$ Calcd.: 304.0701; Found: 304.0704; HPLC (Chiralcel OD-H, hexane:isopropanol = 98:2, flow rate $0.5 \mathrm{~mL} / \mathrm{min}, \lambda=254 \mathrm{~nm}$ ): $t_{\mathrm{R}}=14.7 \mathrm{~min}$ (minor), $t_{\mathrm{R}}=15.3 \mathrm{~min}$ (major).

4-Methoxy- $N$-(2-phenylpropyl)aniline, 1f [17]. The product (45 mg, 74\% yield, 83\% ee) was obtained as a colourless oil according to the general procedure in $3 \mathrm{~d}$; ${ }^{1} \mathrm{H}$ NMR $(400 \mathrm{MHz}$, $\left.\mathrm{CDCl}_{3}\right) \delta 1.32(\mathrm{~d}, J=7.0 \mathrm{~Hz}, 3 \mathrm{H}$ ), 3.00-3.08 (m, 1H), 3.19 (dd, A of $\left.\mathrm{ABX}, J_{\mathrm{AB}}=12.2 \mathrm{~Hz}, J_{\mathrm{AX}}=8.3 \mathrm{~Hz}, 1 \mathrm{H}\right), 3.30\left(\mathrm{dd}, \mathrm{B}\right.$ of $\mathrm{ABX}, J_{\mathrm{AB}}=$ $\left.12.2 \mathrm{~Hz}, J_{\mathrm{BX}}=6.1 \mathrm{~Hz}, 1 \mathrm{H}\right), 3.74(\mathrm{~s}, 3 \mathrm{H}), 6.52-6.56(\mathrm{~m}, 2 \mathrm{H})$, 6.74-6.77 (m, 2H), 7.21-7.25 (m, 3H), 7.30-7.35 (m, 2H); ${ }^{13} \mathrm{C}$ NMR (100 MHz, $\left.\mathrm{CDCl}_{3}\right) \delta 19.8,39.2,52.0,55.8,114.4,114.9$, $126.6,127.3,128.7,142.4,144.6,152.1 ; \mathrm{C}_{16} \mathrm{H}_{20} \mathrm{NO}[\mathrm{M}+\mathrm{H}]^{+}: \mathrm{m} / z$ Calcd.: 242.1539; Found: 242.1537; HPLC (Chiralcel OJ, hexane :isopropanol $=90: 10$, flow rate $0.5 \mathrm{~mL} / \mathrm{min}, \lambda=254 \mathrm{~nm}): t_{\mathrm{R}}=$ $19.6 \mathrm{~min}$ (minor), $t_{\mathrm{R}}=23.1 \mathrm{~min}$ (major).

3-Methoxy- $N$-(2-(o-tolyl)propyl)aniline, 1g. The product (31 mg, 49\% yield, $91 \%$ ee) was obtained a pale yellow oil according to the general procedure in $3 \mathrm{~d} ;{ }^{1} \mathrm{H}$ NMR $(400 \mathrm{MHz}$, $\left.\mathrm{CDCl}_{3}\right) \delta 1.21(\mathrm{~d}, J=6.4 \mathrm{~Hz}, 3 \mathrm{H}), 2.24(\mathrm{~s}, 3 \mathrm{H}), 3.18-3.32(\mathrm{~m}, 3 \mathrm{H})$, $3.54(\mathrm{brs}, 1 \mathrm{H}), 3.68(\mathrm{~s}, 3 \mathrm{H}), 6.06(\mathrm{t}, J=2.2 \mathrm{~Hz}, 1 \mathrm{H}), 6.11(\mathrm{dd}, J=$ 8.0, $1.5 \mathrm{~Hz}, 1 \mathrm{H}), 6.18(\mathrm{dd}, J=8.0,2.2 \mathrm{~Hz}, 1 \mathrm{H}), 6.98(\mathrm{t}, J=8.0 \mathrm{~Hz}$, $1 \mathrm{H}), 7.04-7.11(\mathrm{~m}, 2 \mathrm{H}), 7.13-7.15(\mathrm{~m}, 2 \mathrm{H}) ;{ }^{13} \mathrm{C}$ NMR $(100 \mathrm{MHz}$, $\left.\mathrm{CDCl}_{3}\right) \delta 19.6$ (2C), 34.2, 50.2, 55.1, 98.8, 102.5, 106.0, 125.3,
$126.2,126.5,129.9,130.5,136.2,142.6,149.6,160.9 ; \mathrm{C}_{17} \mathrm{H}_{22} \mathrm{NO}$ [M+H] $]^{+} \mathrm{m} / \mathrm{z}$ Calcd.: 256.1701; Found: 256.1703; HPLC (Chiralcel OD-H, hexane:isopropanol = 98:2, flow rate 0.5 $\mathrm{mL} / \mathrm{min}, \lambda=254 \mathrm{~nm}$ ): $t_{\mathrm{R}}=41.3 \mathrm{~min}$ (minor), $t_{\mathrm{R}}=52.7 \mathrm{~min}$ (major).

4-Methoxy- $N$-(2-m-tolylpropyl)aniline, 1h. The product (39 mg, $61 \%$ yield, $80 \%$ ee) was obtained as a pale yellow oil according to the general procedure in $3 \mathrm{~d} ;{ }^{1} \mathrm{H}$ NMR $(400 \mathrm{MHz}$, $\left.\mathrm{CDCl}_{3}\right) \delta 1.31(\mathrm{~d}, J=7.0 \mathrm{~Hz}, 3 \mathrm{H}), 2.35(\mathrm{~s}, 3 \mathrm{H}), 2.97-3.03(\mathrm{~m}, 1 \mathrm{H})$, 3.18 (dd, $A$ of $\left.A B X, J_{\mathrm{AB}}=12.1 \mathrm{~Hz}, J_{\mathrm{AX}}=8.3 \mathrm{~Hz}, 1 \mathrm{H}\right), 3.28(\mathrm{dd}, \mathrm{B}$ of $\left.\mathrm{ABX}, J_{\mathrm{AB}}=12.1 \mathrm{~Hz}, J_{\mathrm{BX}}=6.1 \mathrm{~Hz}, 1 \mathrm{H}\right), 3.74(\mathrm{~s}, 3 \mathrm{H}), 6.53-6.57(\mathrm{~m}$, 2H), 6.74-6.78 (m, 2H), 7.01-7.06 (m, 3H), $7.21(\mathrm{~d}, J=8.2 \mathrm{~Hz}$, $1 \mathrm{H}) ;{ }^{13} \mathrm{C}$ NMR $\left(100 \mathrm{MHz}, \mathrm{CDCl}_{3}\right) \delta 20.0,21.6,39.3,52.1,55.9$, 114.5, 115.0, 124.4, 127.5, 128.1, 128.7, 138.4, 142.5, 144.7, 152.2; HRMS for $\mathrm{C}_{17} \mathrm{H}_{22} \mathrm{NO}[\mathrm{M}+\mathrm{H}]^{+}: \mathrm{m} / z$ Calcd.: 256.1701; Found: 256.1700; HPLC (Chiralcel OD-H, hexane:isopropanol = 99:1, flow rate $0.5 \mathrm{~mL} / \mathrm{min}, \lambda=254 \mathrm{~nm}$ ): $t_{\mathrm{R}}=42.7 \mathrm{~min}$ (major), $t_{\mathrm{R}}=49.9 \mathrm{~min}$ (minor).

3-Methoxy- $N$-(2-( $m$-tolyl)propyl)aniline, 1i. The product (50 mg, 78\% yield, $84 \%$ ee) was obtained as a pale yellow oil according to the general procedure in $3 \mathrm{~d} ;{ }^{1} \mathrm{H}$ NMR $(400 \mathrm{MHz}$, $\left.\mathrm{CDCl}_{3}\right) \delta 1.24(\mathrm{~d}, J=7.0 \mathrm{~Hz}, 3 \mathrm{H}), 2.27(\mathrm{~s}, 3 \mathrm{H}), 2.89-2.98(\mathrm{~m}, 1 \mathrm{H})$, 3.14 (dd, A of ABX, $J_{\mathrm{AB}}=12.3 \mathrm{~Hz}, J_{\mathrm{AX}}=8.3 \mathrm{~Hz}, 1 \mathrm{H}$ ), 3.23 (dd, B of $\left.\mathrm{ABX}, J_{\mathrm{AB}}=12.3 \mathrm{~Hz}, J_{\mathrm{BX}}=6.2 \mathrm{~Hz}, 1 \mathrm{H}\right), 3.53$ (brs, $\left.1 \mathrm{H}\right), 3.68(\mathrm{~s}, 3 \mathrm{H})$, $6.06(\mathrm{t}, J=2.3 \mathrm{~Hz}, 1 \mathrm{H}), 6.11(\mathrm{dd}, J=8.1,1.5 \mathrm{~Hz}, 1 \mathrm{H}), 6.18(\mathrm{dd}, J=$ 8.1, $2.3 \mathrm{~Hz}, 1 \mathrm{H}), 6.94-7.00(\mathrm{~m}, 4 \mathrm{H}), 7.15(\mathrm{t}, J=7.7 \mathrm{~Hz}, 1 \mathrm{H}) ;{ }^{13} \mathrm{C}$ NMR $\left(100 \mathrm{MHz}^{\left.-\mathrm{CDCl}_{3}\right)} \delta 19.3,21.4,38.6,50.9,55.7,101.9\right.$, 103.2, 124.3, 127.4, 128.0, 128.9, 129.9, 130.3, 138.3, 144.5, 149.6, 160.9; $\mathrm{C}_{17} \mathrm{H}_{22} \mathrm{NO}[\mathrm{M}+\mathrm{H}]^{+}: \mathrm{m} / z$ Calcd.: 256.1701; Found: 256.1703; HPLC (Chiralcel OD-H, hexane:isopropanol $=98: 2$, flow rate $0.5 \mathrm{~mL} / \mathrm{min}, \lambda=254 \mathrm{~nm}$ ): $t_{\mathrm{R}}=37.8 \mathrm{~min}$ (minor), $t_{\mathrm{R}}=$ 47.9 min (major).

$N$-(2-m-Tolylpropyl)aniline, 1j. The product (32 mg, 56\% yield, $80 \%$ ee) was obtained as a colourless oil according to the general procedure in $3 \mathrm{~d}$; ${ }^{1} \mathrm{H}$ NMR $\left(400 \mathrm{MHz}, \mathrm{CDCl}_{3}\right) \delta 1.24(\mathrm{~d}, J$ $=7.0 \mathrm{~Hz}, 3 \mathrm{H}), 2.27(\mathrm{~s}, 3 \mathrm{H}), 2.89-2.98(\mathrm{~m}, 1 \mathrm{H}), 3.14(\mathrm{dd}, \mathrm{A}$ of $\left.\mathrm{ABX}, J_{\mathrm{AB}}=12.1 \mathrm{~Hz}, J_{\mathrm{AX}}=8.3 \mathrm{~Hz}, 1 \mathrm{H}\right), 3.24\left(\mathrm{dd}, \mathrm{B}\right.$ of $\mathrm{ABX}, J_{\mathrm{AB}}=$ $\left.12.2 \mathrm{~Hz}, J_{\mathrm{BX}}=6.2 \mathrm{~Hz}, 1 \mathrm{H}\right), 3.49(\mathrm{brs}, 1 \mathrm{H}), 6.49(\mathrm{dd}, J=8.4,0.9 \mathrm{~Hz}$, $2 \mathrm{H}), 6.61(\mathrm{t}, J=7.3 \mathrm{~Hz}, 1 \mathrm{H}), 6.96(\mathrm{t}, J=8.4 \mathrm{~Hz}, 3 \mathrm{H}), 7.08-7.12$ (m, 2H), 7.14-7.16 (m, 1H); ${ }^{13} \mathrm{C}$ NMR (100 MHz, $\left.\mathrm{CDCl}_{3}\right) \delta 19.9$, 21.5, 39.2, 50.9, 113.0, 117.3, 124.3, 127.4, 128.0, 128.6, 129.3, 138.3, 144.5, 148.2; MS (CI) for $\mathrm{C}_{16} \mathrm{H}_{20} \mathrm{~N}[\mathrm{M}+\mathrm{H}]^{+}: \mathrm{m} / z 226$ (100\%); Anal. Calcd. for $\mathrm{C}_{16} \mathrm{H}_{19} \mathrm{~N}$ : C, 85.28; H, 8.50; N, 6.22. Found: C, 84.71; H, 8.83; N, 6.42. HPLC (Chiralcel OD-H, hexane:isopropanol $=99.5: 0.5$, flow rate $4 \mathrm{~mL} / \mathrm{min}, \lambda=254$ $\mathrm{nm}): t_{\mathrm{R}}=11.3 \mathrm{~min}$ (minor), $t_{\mathrm{R}}=12.3 \mathrm{~min}$ (major).

$N$-(2-(4-Methoxyphenyl)propyl)-4-methylaniline, 1k. The product (39 mg, 61\% yield, $82 \%$ ee) was obtained as a colourless oil according to the general procedure in $3 \mathrm{~d} ;{ }^{1} \mathrm{H}$ NMR (400 MHz, $\left.\mathrm{CDCl}_{3}\right) \delta 1.29(\mathrm{~d}, J=6.9 \mathrm{~Hz}, 3 \mathrm{H}), 2.23(\mathrm{~s}, 3 \mathrm{H})$, 2.96-3.04 (m, 1H), 3.15 (dd, A of ABX, $J_{\mathrm{AB}}=12.2 \mathrm{~Hz}, J_{\mathrm{AX}}=8.4 \mathrm{~Hz}$, $1 \mathrm{H}), 3.29$ (dd, B of $\left.A B X, J_{A B}=12.2 \mathrm{~Hz}, J_{B X}=6.0 \mathrm{~Hz}, 1 \mathrm{H}\right), 3.80(\mathrm{~s}$, $3 \mathrm{H}), 6.48-6.51(\mathrm{~m}, 2 \mathrm{H}), 6.85-6.88(\mathrm{~m}, 2 \mathrm{H}), 6.97(\mathrm{~d}, J=8.4 \mathrm{~Hz}$, 2H), 7.12-7.16 (m, 2H); ${ }^{13} \mathrm{C} \mathrm{NMR}\left(100 \mathrm{MHz}, \mathrm{CDCl}_{3}\right) \delta 20.0,21.2$, 38.9, 51.5, 55.9, 114.0, 114.1, 126.5, 128.2, 129.8, 136.6, 145.9, 158.2, 158.3; HRMS for $\mathrm{C}_{17} \mathrm{H}_{22} \mathrm{NO}[\mathrm{M}+\mathrm{H}]^{+}: \mathrm{m} / z$ Calcd.: 256.1701; Found: 256.1700; HPLC (Chiralcel OD-H, 
hexane:isopropanol $=98.5: 1.5$, flow rate $0.5 \mathrm{~mL} / \mathrm{min}, \lambda=254$ $\mathrm{nm}): t_{\mathrm{R}}=19.9 \min$ (minor), $t_{\mathrm{R}}=22.2 \mathrm{~min}$ (major).

4-Bromo- $N$-(2-(4-methoxyphenyl)propyl)aniline, 11. The product ( $41 \mathrm{mg}, 51 \%$ yield, $84 \%$ ee) was obtained as a pale yellow oil according to the general procedure in $3 \mathrm{~d} ;{ }^{1} \mathrm{H}$ NMR (400 MHz, $\left.\mathrm{CDCl}_{3}\right) \delta 1.30(\mathrm{~d}, J=6.9 \mathrm{~Hz}, 3 \mathrm{H}), 2.94-3.03(\mathrm{~m}, 1 \mathrm{H})$, 3.13 (dd, A of ABX, $J_{\mathrm{AB}}=12.2 \mathrm{~Hz}, J_{\mathrm{AX}}=8.6 \mathrm{~Hz}, 1 \mathrm{H}$ ), 3.28 (dd, B of $\left.\mathrm{ABX}, J_{\mathrm{AB}}=12.2 \mathrm{~Hz}, J_{\mathrm{BX}}=6.0 \mathrm{~Hz}, 1 \mathrm{H}\right), 3.80(\mathrm{~s}, 3 \mathrm{H}), 6.41-6.45(\mathrm{~m}$, $2 \mathrm{H}), 6.86-6.88(\mathrm{~m}, 2 \mathrm{H}), 7.11-7.14(\mathrm{~m}, 2 \mathrm{H}), 7.20-7.24(\mathrm{~m}, 2 \mathrm{H})$; ${ }^{13} \mathrm{C}$ NMR $\left(100 \mathrm{MHz}, \mathrm{CDCl}_{3}\right) \delta 20.3,38.7,51.4,55.7,109.2,114.5$, $114.9,128.5,132.3,136.6,147.5,158.8$; HRMS for $\mathrm{C}_{16} \mathrm{H}_{19} \mathrm{BrNO}$ $[\mathrm{M}+\mathrm{H}]^{+}: \quad \mathrm{m} / \mathrm{z}$ Calcd.: 320.0650; Found: 320.0659; HPLC (Chiralcel OD-H, hexane:isopropanol $=98: 2$, flow rate 0.5 $\mathrm{mL} / \mathrm{min}, \lambda=254 \mathrm{~nm}$ ): $t_{\mathrm{R}}=23.0 \mathrm{~min}$ (minor), $t_{\mathrm{R}}=27.3 \mathrm{~min}$ (major).

4-Methoxy- $N$-(2-(4-(trifluoromethyl)phenyl)propyl)aniline, 1m. The product (54 mg, $70 \%$ yield, $81 \%$ ee) was obtained as a colourless oil according to the general procedure in $3 \mathrm{~d} ;{ }^{1} \mathrm{H}$ NMR (400 MHz, $\left.\mathrm{CDCl}_{3}\right) \delta 1.34(\mathrm{~d}, J=6.9 \mathrm{~Hz}, 3 \mathrm{H}), 3.08-3.17(\mathrm{~m}$, $1 \mathrm{H}$ ), 3.22 (dd, A of ABX, $J_{\mathrm{AB}}=12.4 \mathrm{~Hz}, J_{\mathrm{AX}}=8.2 \mathrm{~Hz}, 1 \mathrm{H}$ ), 3.33 (dd, $\mathrm{B}$ of $\left.\mathrm{ABX}, J_{\mathrm{AB}}=12.4 \mathrm{~Hz}, J_{\mathrm{BX}}=6.0 \mathrm{~Hz}, 1 \mathrm{H}\right), 3.75(\mathrm{~s}, 3 \mathrm{H}), 6.52-6.56$ (m, 2H), 6.75-6.79 (m, 2H), $7.33(\mathrm{~d}, J=8.1 \mathrm{~Hz}, 2 \mathrm{H}), 7.58(\mathrm{~d}, J=$ $8.1 \mathrm{~Hz}, 2 \mathrm{H}) ;{ }^{13} \mathrm{C}$ NMR $\left(100 \mathrm{MHz}, \mathrm{CDCl}_{3}\right) \delta 19.5,39.2,51.8,55.8$, $114.5,115.0,124.3\left(\mathrm{q}, J_{\mathrm{CF}}=268.9 \mathrm{~Hz}\right) 125.6\left(\mathrm{q}, J_{\mathrm{CF}}=3.6 \mathrm{~Hz}\right)$, 127.8, 128.9 (q, $J_{\mathrm{CF}}=21.1 \mathrm{~Hz}$ ), 141.9, 148.9, 152.3; HRMS for $\mathrm{C}_{16} \mathrm{H}_{19} \mathrm{BrNO}[\mathrm{M}+\mathrm{H}]+: \mathrm{m} / z$ Calcd.: 310.1419; Found: 310.1419; HPLC (Chiralcel OD-H, hexane:isopropanol = 98:2, flow rate 0.5 $\mathrm{mL} / \mathrm{min}, \lambda=254 \mathrm{~nm}$ ): $t_{\mathrm{R}}=27.1 \mathrm{~min}$ (major), $t_{\mathrm{R}}=29.9 \mathrm{~min}$ (minor).

$N$-(2-(4-(Trifluoromethyl)phenyl)propyl)aniline, 1n. The product (39 $\mathrm{mg}, 56 \%$ yield, $79 \%$ ee) was obtained as a colourless oil according to the general procedure in $3 \mathrm{~d} ;{ }^{1} \mathrm{H}$ NMR (400 MHz, $\left.\mathrm{CDCl}_{3}\right) \delta 1.35(\mathrm{~d}, J=6.9 \mathrm{~Hz}, 3 \mathrm{H}), 3.09-3.18(\mathrm{~m}$, $1 \mathrm{H}), 3.26\left(\mathrm{dd}, \mathrm{A}\right.$ of $\mathrm{ABX}, J_{\mathrm{AB}}=12.6 \mathrm{~Hz}, J_{\mathrm{AX}}=8.3 \mathrm{~Hz}, 1 \mathrm{H}$ ), 3.37 (dd, $\mathrm{B}$ of $\left.\mathrm{ABX}, J_{\mathrm{AB}}=12.6 \mathrm{~Hz}, J_{\mathrm{BX}}=6.2 \mathrm{~Hz}, 1 \mathrm{H}\right), 3.52$ (brs, $\left.1 \mathrm{H}\right)$, 6.56-6.59 (m, 2H), 6.64-6.72 (m, 1H), 7.14-7.19 (m, 2H), 7.33 $(\mathrm{d}, J=8.1 \mathrm{~Hz}, 2 \mathrm{H}), 7.58(\mathrm{~d}, J=8.1 \mathrm{~Hz}, 2 \mathrm{H}) ;{ }^{13} \mathrm{C} \mathrm{NMR}(100 \mathrm{MHz}$, $\left.\mathrm{CDCl}_{3}\right) \delta 19.6,38.6,50.7,112.9,117.6,125.6\left(\mathrm{q}, J_{\mathrm{CF}}=3.8 \mathrm{~Hz}\right)$, 127.6, 129.0 (q, JCF $=30.6 \mathrm{~Hz}$ ), 129.3, 147.7, 148.7 (The carbon resonance $\mathrm{CF}_{3}$ was not observed, possibly due to overlap with other aromatic carbon resonances); $\mathrm{C}_{16} \mathrm{H}_{17} \mathrm{~F}_{3} \mathrm{~N}[\mathrm{M}+\mathrm{H}]^{+}: \mathrm{m} / z$ Calcd.: 280.1313; Found: 280.1307; HPLC (Chiralcel OD-H, hexane:isopropanol $=98: 2$, flow rate $0.5 \mathrm{~mL} / \mathrm{min}, \lambda=254 \mathrm{~nm})$ : $t_{\mathrm{R}}=29.3 \mathrm{~min}$ (minor), $t_{\mathrm{R}}=33.7 \mathrm{~min}$ (major).

4-Methoxy- $N$-(2-(naphthalen-2-yl)propyl)aniline, 1p [17]. The product ( $59 \mathrm{mg}, 81 \%$ yield, $83 \%$ ee) was obtained as a pale yellow oil according to the general procedure in $3 \mathrm{~d} ;{ }^{1} \mathrm{H}$ NMR (400 MHz, $\left.\mathrm{CDCl}_{3}\right) \delta 1.41(\mathrm{~d}, J=6.8 \mathrm{~Hz}, 3 \mathrm{H}), 3.18-3.26(\mathrm{~m}, 1 \mathrm{H})$, 3.29 (dd, A of ABX, $J_{\mathrm{AB}}=12.0 \mathrm{~Hz}, J_{\mathrm{AX}}=8.4 \mathrm{~Hz}, 1 \mathrm{H}$ ), 3.39 (dd, B of $\left.\mathrm{ABX}, J_{\mathrm{AB}}=12.0 \mathrm{~Hz}, J_{\mathrm{BX}}=5.7 \mathrm{~Hz}, 1 \mathrm{H}\right), 3.74(\mathrm{~s}, 3 \mathrm{H}), 6.52-6.56(\mathrm{~m}$, 2H), 6.74-6.78 (m, 2H), 7.37 (dd, $J=8.5,1.7 \mathrm{~Hz}, 1 \mathrm{H}), 7.43-7.50$ $(\mathrm{m}, 2 \mathrm{H}), 7.66(\mathrm{~s}, 1 \mathrm{H}), 7.81(\mathrm{t}, J=8.5 \mathrm{~Hz}, 3 \mathrm{H}) ;{ }^{13} \mathrm{C} \mathrm{NMR}(100 \mathrm{MHz}$, $\left.\mathrm{CDCl}_{3}\right) \delta 19.9,39.4,51.8,55.8,114.4,114.9,125.5,125.9,126.1$, 127.6(2), 127.6(4), 128.4, 130.2, 132.5, 133.6, 142.0, 142.3, 152.1; HRMS for $\mathrm{C}_{20} \mathrm{H}_{22} \mathrm{NO}[\mathrm{M}+\mathrm{H}]^{+}: \mathrm{m} / z$ Calcd: 292.1701; Found: 292.1711; HPLC (Chiralcel OD-H, hexane:isopropanol = 98:2, flow rate $0.5 \mathrm{~mL} / \mathrm{min}, \lambda=254 \mathrm{~nm}$ ): $t_{\mathrm{R}}=36.4 \mathrm{~min}$ (major),
$t_{\mathrm{R}}=39.3 \mathrm{~min}$ (minor).

\section{Results and discussion}

Hydroformylation usually affords a linear aldehyde. However, it is the branched aldehyde that could lead to a chiral amine. Hence, in order to turn the product of hydroaminomethylation into highly optically active, one must first maximise the $i / n$ selectivity of the transformation (Scheme 2). $\mathrm{Li}$ and co-workers [16] reported the selective hydroformylation of styrene to the corresponding $\alpha$-branched aldehyde with a $\mathrm{Rh}$ catalyst containing a triarylphosphine ligand possessing a long-chain alkoxy group. The reaction takes place in toluene, with $0.2 \mathrm{~mol} \%$ [Rh(acac)(CO) 2 ] and $0.4 \mathrm{~mol} \%$ phosphine ligand at 15 bar syngas and $50{ }^{\circ} \mathrm{C}$. As a starting point, we decided to use these conditions for our hydroaminomethylation sequence, with $p$-anisidine as the model amine. A range of phosphine ligands were initially tested. Disappointedly, we quickly found that although the Rh-phosphine catalyst promoted the hydroformylation of styrene and the enamine was formed, hydrogenation of the latter was never observed, even in the presence of an acid (Scheme 1). This may not be surprising as one of the main problems with the hydroaminomethylation protocols is the slow hydrogenation of the enamine/imine intermediate [9].

Knowing that the imine hydrogenation could be effected by other catalysts, we then focused on the idea of using two different catalysts, one for the hydroformylation and the other for the hydrogenation step. In particular, List et al. [17] had developed a protocol on asymmetric reductive amination of $\alpha$-branched aldehydes via DKR, in which a chiral phosphoric acid acted as the organocatalyst and a Hantzsch ester (HEH) as the hydrogen source. This became our choice to effect the reductive amination step.

We combined the $\left[\mathrm{Rh}(\mathrm{acac})(\mathrm{CO})_{2}\right]$ catalyst precursor with $\mathrm{PPh}_{3}, \mathrm{Et}-\mathrm{HEH}$ and the phosphoric acid TRIP in toluene to examine the reaction of $p$-methylstyrene with $p$-anisidine at 11 bar syngas and $50{ }^{\circ} \mathrm{C}$ (Scheme 3 ). The reaction mixture was left stirring for $2 \mathrm{~d}$. Delightfully, after flash chromatography purification, a promising $41 \%$ isolated yield was obtained for the desired product 1 a with a good enantioselectivity of $81 \%$ ee.

Encouraged by this result, optimisation of the conditions was then undertaken, aiming to improve the yield and enantioselectivity. First, the effect of the HEH was studied. The results are shown in Table 1 . The reaction takes place under a syngas pressure; therefore $\mathrm{H}_{2}$ could act as the reductant for the hydrogenation step. However, the presence of HEH is essential in this reaction, suggesting that the hydrogen source for the reductive amination or more precisely the reduction of the imino bond comes from the HEH (entry 1). An excess of Et-HEH provides a positive effect on the yield (entry 2 vs entry 3 ). Whilst a bulkier $t \mathrm{Bu}$-HEH afforded an increase in the enantioselectivity of the reductive amination of $\alpha$-branched aldehydes [17], very low yield was obtained in our case (entry 5). A decrease in the activity with bulkier HEH's was also observed by List et al. [17]. The effect of the temperature was next examined. Although better enantioselectivities were obtained at a low temperature of $6{ }^{\circ} \mathrm{C}$ for the reductive amination step [17], the hydroam- 


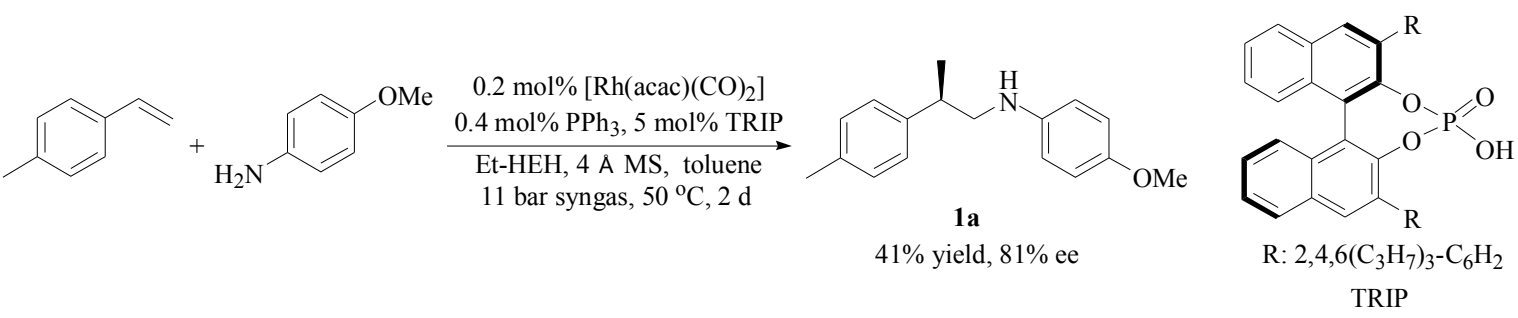

Scheme 3. Preliminary study of the metal- and organo-catalysed asymmetric hydroaminomethylation.

inomethylation in question became much slower when the temperature was dropped to $25{ }^{\circ} \mathrm{C}(3 \mathrm{~d}, 24 \%$ yield, $83 \%$ ee). As maybe expected, a higher temperature of $80{ }^{\circ} \mathrm{C}$ led to a higher yield but a slight decrease in enantioselectivity $(2 \mathrm{~d}, 55 \%$ yield, $79 \%$ ee). Thus, a temperature of $50{ }^{\circ} \mathrm{C}$ was chosen which offered good enantioselectivities whilst maintaining a reasonable rate of reaction.

Table 2 shows the effect of phosphine ligands in this asymmetric hydroaminomethylation. Monophosphine ligands are in general superior compared to diphosphines (entries 1-3 vs 4-8). Within the derivatives of $\mathrm{PPh}_{3}$, a more electron-donating substituent in the ligand leads to an increase in yield (entry 8), whilst an electron-deficient substituent has a negative effect on the yield (entry 7). The increase in yield stems from a higher selectivity for the branched aldehyde in the hydroformylation step. Moser and co-workers [18] showed that $p$-electron-donating groups in the phosphine increase the basicity of the phosphine and the selectivity for $\alpha$-branched aldehydes.

With the outcome of optimisation in hand, we then explored the scope of the methodology using a range of styrenes and anilines. Table 3 shows the effect of substituents at the aniline on the hydroaminomethylation of $p$-methylstyrene. All the products 1a-e were obtained with good enantioselectivity (78\%-86\% ee). Better enantioselectivities were obtained when more sterically-hindered anilines were used (entry 1 vs 2, entry 3 vs 4), and this observation was previously noted in the

Table 1

Effect of Hantzsch esters on the model asymmetric hydroaminomethylation.

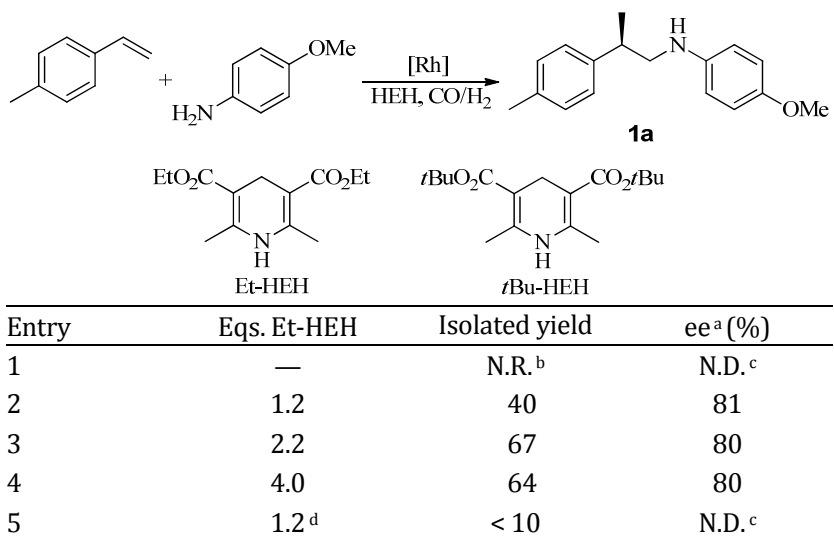

Reaction conditions: $0.4 \mathrm{mmol} p$-methylstyrene, $0.25 \mathrm{mmol} p$-anisidine, $1.25 \mu \mathrm{mol}$ [Rh(acac)(CO) 2 ], $2.5 \mu \mathrm{mol} \mathrm{PPh}_{3}$, Et-HEH (unless otherwise specified), $12.5 \mu \mathrm{mol}$ TRIP, $100 \mathrm{mg} 4 \AA$ MS, $4 \mathrm{~mL}$ toluene, $11 \mathrm{bar} \mathrm{CO} / \mathrm{H}_{2}$ $1: 1,50{ }^{\circ} \mathrm{C}, 2 \mathrm{~d}$.

a Determined by HPLC. ${ }^{\mathrm{b}}$ No reaction. ${ }^{\mathrm{c}}$ Not determined. $\mathrm{d} t \mathrm{Bu}-\mathrm{HEH}$ used. asymmetric reductive amination of ketones [19,20]. Lower yield was obtained when using electron-deficient $p$-bromoaniline (entry 5), reflecting presumably the difficulty encountered in the aldehyde-amine condensation step. This was also observed by List et al. [17] in the organocatalytic reductive amination of $\alpha$-branched aldehydes via DKR and in transition metal- [2] and organo-catalysed [21,22] asymmetric reductive amination of ketones.

We next investigated the asymmetric hydroaminomethylation of different derivatives of styrene with aniline and its analogues (Table 4). Good yields and enantioselectivities were obtained in general. A lower yield was obtained when using an ortho-substituted styrene (entry 2 ). This is due to the $i / n$ selectivity in the hydroformylation step being lower as a result of steric effects, with the ortho substituent inhibiting the formation of the benzylic Rh species that would favour producing the branched aldehyde [23,24]. In fact, when hydroformylation of $p$-methyl and $o$-methylstyrene was compared, the $i / n$ selectivity decreased from 13:1 to 7:1. Similar to what is shown in Table 3, electron-deficient groups in the aniline ring have a detrimental effect on the yield (Table 4, entries 7 and 10). As mentioned, this is likely to result from an inefficient condensation step $[17,22]$. The same could be expected from electron-deficient groups in the styrene ring (entries 8-10) [17].

Table 2

Effect of ligands on the model asymmetric hydroaminomethylation.

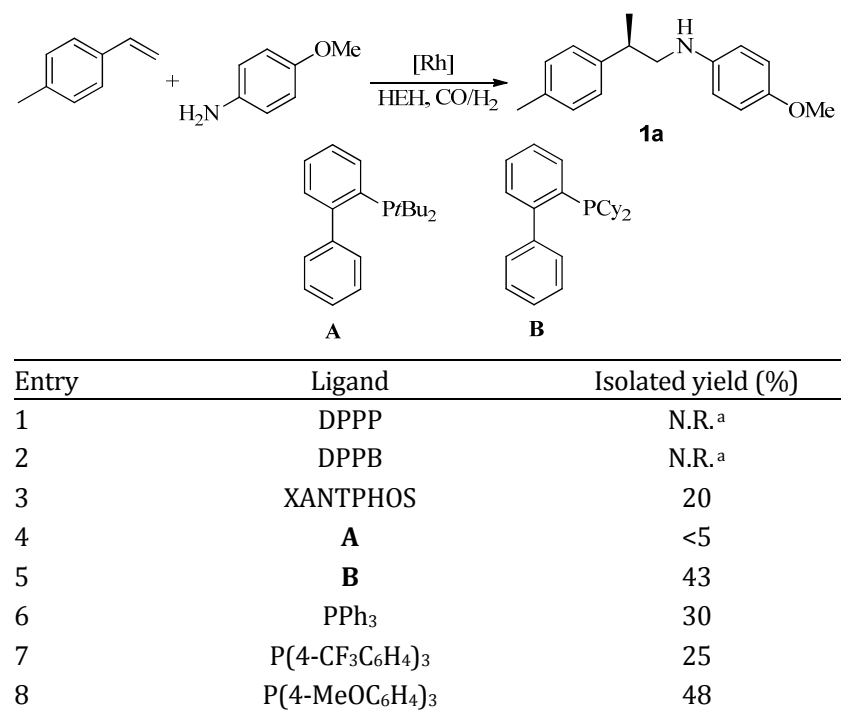

Reactions conditions: the same as those in Table 1 except with different phosphine ligand, $0.6 \mathrm{mmol}$ Et-HEH and $17 \mathrm{~h}$ reaction time.

a No desired reaction; only linear product observed. 
Table 3

Asymmetric hydroaminomethylation of $p$-methylstyrene with different anilines.

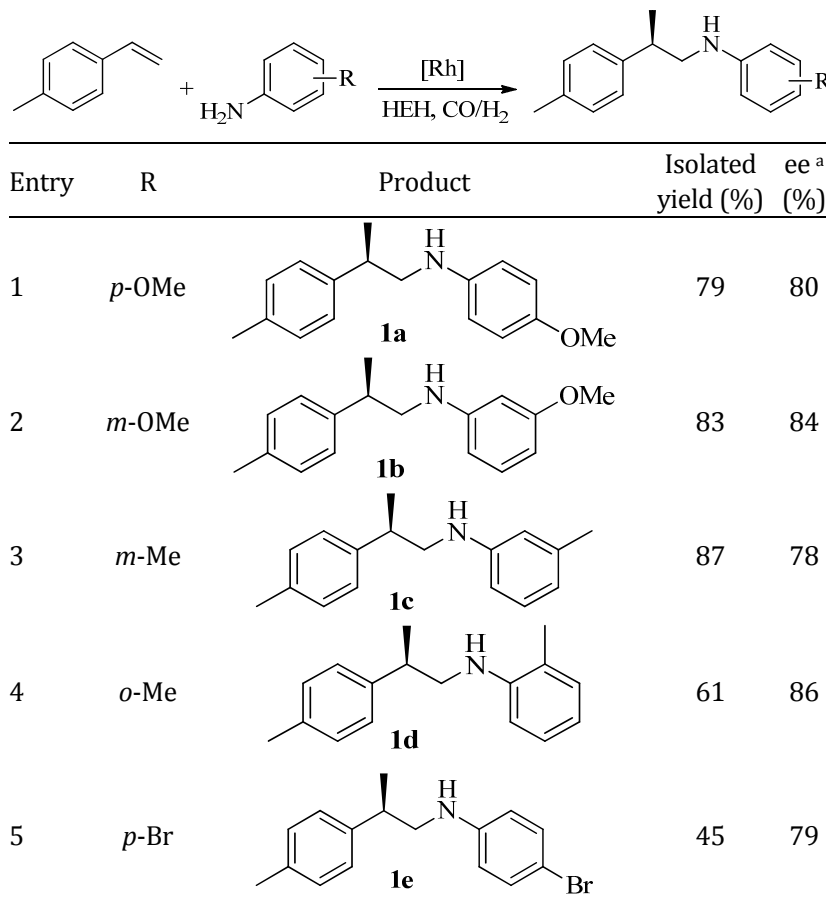

Reactions conditions: the same as those in Table 1 except with 0.25 mmol aniline derivative, $2.5 \mu \mathrm{mol} \mathrm{P}\left(4-\mathrm{CH}_{3} \mathrm{OC}_{6} \mathrm{H}_{4}\right)_{3}, 0.6 \mathrm{mmol} \mathrm{Et}-\mathrm{HEH}$ and $3 \mathrm{~d}$ reaction time.

a Determined by HPLC.

\section{Conclusions}

We have developed a new protocol that enables asymmetric hydroaminomethylation of styrenes, affording $\beta$-chiral amines with good yields and enantioselectivities. To the best of our knowledge, this is the first example of an asymmetric version of this tandem reaction where significant enantioselectivities have been achieved. The transformation is made possible by combining metal- and organo-catalysis, with the former catalysing the hydroformylation while the latter reductive amination via DKR. The protocol provides an attractive pathway for the synthesis of $\beta$-chiral amines, as they can be obtained in one step from easily available starting materials. The main drawback of the protocol is the use of HEH as hydrogen source. A single chiral metal complex to catalyse both steps will be more desirable, and lead to a greener procedure with $\mathrm{H}_{2}$ as the only hydrogen source.

\section{References}

[1] Li C Q, Wang C, Villa-Marcos B, Xiao J L. J Am Chem Soc, 2008, 130 : 14450

[2] Li C Q, Villa-Marcos B, Xiao J L. J Am Chem Soc, 2009, 131: 6967

[3] Villa-Marcos B, Li C Q, Mulholland K R, Hogan P J, Xiao J L. Molecules, 2010, 15: 2453

[4] Tang WJ, Johnston S, Iggo J A, Berry N G, Phelan M, Lian L Y, Bacsa J, Xiao J L. Angew Chem Int Ed, 2013, 52: 1668

[5] Tang W J, Johnston S, Li C Q, Iggo J A, Bacsa J, Xiao J L. Chem Eur J,
Table 4

Asymmetric hydroaminomethylation of different derivatives of styrene.

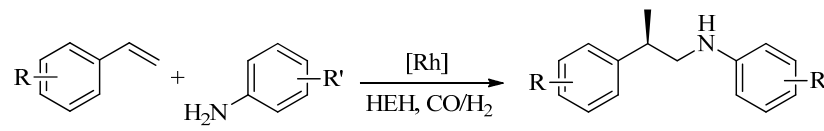

\begin{tabular}{llllll}
\hline Entry & $\mathrm{R}$ & $\mathrm{R}^{\prime}$ & Product & $\begin{array}{c}\text { Isolated } \\
\text { yield (\%) }\end{array}$ & $(\%)$ \\
\hline
\end{tabular}

12

$2 \quad o$-Me $m$-OM<smiles>COc1cccc(NCC(C)c2ccccc2C)c1</smiles>

$3 \quad m$-Me $p$-OMe<smiles>COc1ccc(NCC(C)c2cccc(C)c2)cc1</smiles>
$m$-Me $m$-OM<smiles>COc1cccc(NCC(C)c2cccc(C)c2)c1</smiles>

$5 \quad m-\mathrm{Me} \quad \mathrm{H}$<smiles>Cc1cccc([C@H](C)CNc2ccccc2)c1</smiles>

$6 \quad p$-OMe $p$-Me<smiles>C=IC(CNc1ccc(C)cc1)[C@H](I)c1ccc(OC)cc1</smiles>

$p$-OMe $p$-Br<smiles>COc1ccc(C(C)CNc2ccc(Br)cc2)cc1</smiles>
$p-\mathrm{CF}_{3} p-\mathrm{OMe}$<smiles>FC(F)(F)c1ccccc1</smiles><smiles>COc1ccc(NCC(C)c2ccccc2)cc1</smiles>

9

$p-\mathrm{CF}_{3} \quad \mathrm{H}$<smiles>C[C@H](CNc1ccccc1)c1ccc(C(F)(F)F)cc1</smiles>

10
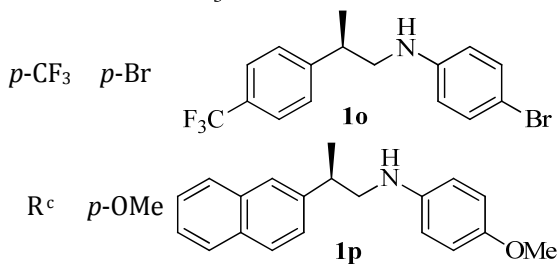<smiles>CNc1ccc(OC)cc1</smiles>

Reaction conditions: the same as those in Table 1 except with $0.4 \mathrm{mmo}$ styrene derivative, $0.25 \mathrm{mmol}$ aniline derivative, $2.5 \mu \mathrm{mo}$ $\mathrm{P}\left(4-\mathrm{CH}_{3} \mathrm{OC}_{6} \mathrm{H}_{4}\right)_{3}, 0.6 \mathrm{mmol}$ Et-HEH and $3 \mathrm{~d}$ reaction time.

a Determined by HPLC.

${ }^{b}$ Not determined.

c2-Vinylnaphthalene (R: 3,4- $\mathrm{C}_{4} \mathrm{H}_{4}$ ) used.

2013, 19: 14187

[6] Van Leeuwen P W N M, Claver C. Rhodium Catalyzed Hydroformylation. Netherlands: Springer, 2000

[7] Marcelli T, Hammar P, Himo F. Adv Synth Catal, 2009, 351: 525

[8] Eilbracht P, Barfacker L, Buss C, Hollmann C, Kitsos-Rzychon B E, Kranemann C L, Rische T, Roggenbuck R, Schmidt A. Chem Rev, 1999, 99: 3329

[9] Ahmed M, Seayad A M, Jackstel R, Beller M. J Am Chem Soc, 2003, 125: 10311 


\section{Graphical Abstract}

Chin. J. Catal., 2015, 36: 106-112 doi: 10.1016/S1872-2067(14)60246-1

\section{Metal and organo-catalysed asymmetric hydroaminomethylation of styrenes}

Barbara Villa-Marcos, Jianliang Xiao*

University of Liverpool, $U K$

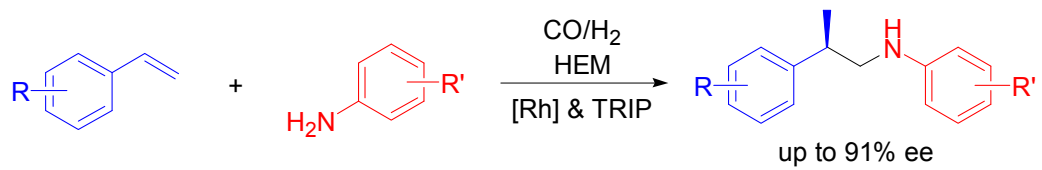

Combining metal-catalysed hydroformylation with a chiral organo-acid-catalysed reductive amination allows for asymmetric hydroaminomethylation to be realised.

[10] Reppe W, Vetter H. Liebigs Ann Chem, 1953, 582: 133

[11] Crozet D, Urrutigoity M, Kalck P. ChemCatChem, 2011, 3: 1102

[12] Wu L P, Fleischer I, Jackstell R, Beller M. J Am Chem Soc, 2013, 135 : 3989

[13] Gülak S, Wu L P, Liu Q, Franke R, Jackstell R, Beller M. Angew Chem Int Ed, 2014, 53: 7320

[14] Crozet D, Kefalidis C E, Urrutigoïty M, Maron L, Kalck P. ACS Catal, 2014, 4: 435

[15] Noonan G M, Newton D, Cobley C J, Suárez A, Pizzano A, Clarke M L. Adv Synth Catal, 2010, 352: 1047

[16] Guo Y, Fu H Y, Chen H, Li X J. Catal Commum, 2008, 9: 1842

[17] Hoffmann S, Nicoletti M, List B. J Am Chem Soc, 2006, 128: 13074

[18] Moser W R, Papile C J, Brannon D A, Duwell R A, Weininger S J. J Mol Catal, 1987, 41: 271
[19] Mrsic N, Minnaard A J, Feringa B L, de Vries J G. J Am Chem Soc, 2009, 131: 8358

[20] Rubio-Pérez L, Pérez-Flores F J, Sharma P, Velasco L, Cabrera A. Org Lett, 2009, 11: 265

[21] Hoffmann S, Seayad A M, List B. Angew Chem Int Ed, 2005, 44: 7424

[22] Storer R I, Carrera D E, Ni Y, MacMillan D W C. J Am Chem Soc, 2006, 128: 84

[23] Yu S C, Chie Y M, Guan Z H, Zou Y P, Li W, Zhang X. Org Lett, 2009, 11: 241

[24] Van Leeuwen P W N M, Kamer P C J, Reek J N H, Dierkes P. Chem Rev, 2000, 100: 2741

[25] Wang C, Pettman A, Basca J, Xiao J L. Angew Chem Int Ed, 2010, 49: 7548 